\section{JTI}

JOURNAL OF

TRAUMA AND INJURY

Received: June 16, 2019

Revised: August 23, 2019

Accepted: August 30, 2019

\section{Correspondence to}

Seok Chan Eun, M.D., Ph.D.

Department of Plastic and Reconstructive Surgery, Seoul National University Bun-

dang Hospital, Seoul National University

College of Medicine, 82 Gumi-ro 173

beon-gil, Bundang-gu, Seongnam 13620,

Korea

Tel: $+82-31-787-7223$

Fax: +82-31-787-4055

E-mail:seokchan.eun@gmail.com

\title{
Free Flap Reconstruction in Patients with Traumatic Injury of the Forefoot
}

\author{
Shin Hyuk Kang, M.D., Ph.D., Jeongseok Oh, M.D., Seok Chan Eun, M.D., Ph.D. \\ Department of Plastic and Reconstructive Surgery, Seoul National University Bundang \\ Hospital, Seoul National University College of Medicine, Seongnam, Korea
}

Many techniques have been developed for reconstruction of the hand; however, less attention has been paid to foot reconstruction techniques. In particular, reconstruction of the forefoot and big toe has been considered a minor procedure despite the importance of these body parts for standing and walking. Most of the weight load on the foot is concentrated on the forefoot and big toe, whereas the other toes have a minor role in weight bearing. Moreover, the forefoot and big toe are important for maintaining balance and supporting the body when changing directions. Recently, attention has been focused on the aesthetic appearance and functional aspects of the body, which are important considerations in the field of reconstructive surgery. In patients for whom flap reconstruction in the forefoot and big toe is planned, clinicians should pay close attention to flap survival as well as functional and cosmetic outcomes of surgery. In particular, it is important to assess the ability of the flap to withstand functional weight bearing and maintain sufficient durability under shearing force. Recovery of protective sensation in the forefoot area can reduce the risk of flap loss and promote rapid rehabilitation and functional recovery. Here, we report our experience with two cases of successful reconstruction of the forefoot and big toe with a sensate anterolateral thigh flap, with a review of the relevant literature.

Keywords: Forefoot; Hallux; Functional; Aesthetic; Reconstruction

\section{INTRODUCTION}

Severe trauma and injury of the foot are less frequent than those of the hand, and the appropriate reconstructive methods remain unclear and difficult to execute. Previous studies have recommended various functional and cosmetic reconstruction methods for hand injuries [1,2], while foot injuries are often more severe and clinicians face the 


\section{JTI}

challenge of preserving the limb function. The forefoot is defined as the portion of the foot inferior to the tarsometatarsal joints and is composed of tendons, ligaments, soft tissue, and 19 bones. Together with the midfoot and hindfoot, the forefoot is one of the primary regions of the foot and is considered an important weight-bearing body part. Severe damage to the forefoot is frequently associated with damage to the skin, soft tissue, as well as blood vessels, nerves, tendons, and bone [3,4]. Moreover, inadequate reconstruction can lead to severe restrictions in standing and walking. Among the components of the forefoot area, the big toe is considered the most important part. However, active reconstruction of the big toe is rarely performed. Reconstruction of the forefoot and big toe remains a major challenge in surgical practice, especially in terms of appearance and function. In patients indicated for active reconstruction with flap surgery, clinicians should consider the survival of the flap and the improvement of cosmetic and functional outcomes of surgery. In particular, in the reconstruction of a forefoot with a degloving injury, a flap with relative thinness and appropriate shape can achieve excellent aesthetic results because of the small defect volume. Inclusion of the sensory nerve in the flap can reduce the risk of flap loss and provide rapid rehabilitation and functional recovery. Here, we report on two cases of reconstruction of the forefoot (in a 51-year-old male patient with extensive injury to the right forefoot) and big toe (in a 41-year-old male patient with crushing injury to the big toe) with a sensate anterolateral thigh flap, for which satisfactory functional and cosmetic outcomes were achieved.

\section{CASE REPORT}

\section{Case 1}

The patient was a 51-year-old man with a history of degloving of the right foot due to accidental entrapment in a plant roller during the course of his work (Fig. 1A). $\mathrm{He}$ also sustained fracture of the thoracic ribs at multiple levels with hemothorax development. He underwent emergency operation in the department of thoracic surgery. In department of plastic and reconstructive surgery, osteotomy of the distal bone was performed because of fracture and necrosis of the foot, and stripped skin tissue was applied as a temporary biologic dressing (Fig. 1B). On the 7th day of skin application, necrosis of the skin was observed while the patient's general condition had improved. An anterolateral thigh free flap transfer with the lateral femoral cutaneous nerve (for a sensate flap) was planned. In the evaluation at that time, the patient had a relatively healthy status without underlying diseases such as diabetes and vascular occlusion. With the patient under general anesthesia and in the supine position, debridement of the necrotic skin was performed, which resulted in exposure of the bone. In addition, marginal resection of proximal soft tissue was performed up to the level of healthy tissue presenting skin bleeding. The dorsalis pedis artery and vena comitans were prepared for anastomosis, and the deep peroneal nerve was dissected for a sensory anastomosis. The lateral circumflex femoral artery perforator branches were identified using a handheld Doppler ultrasound device. Subsequently, an anterolateral thigh flap of size $35 \times 15 \mathrm{~cm}$, which was slightly larger than the circumference of the contralateral foot, was elevated (Fig. 1C). After confirming continuity
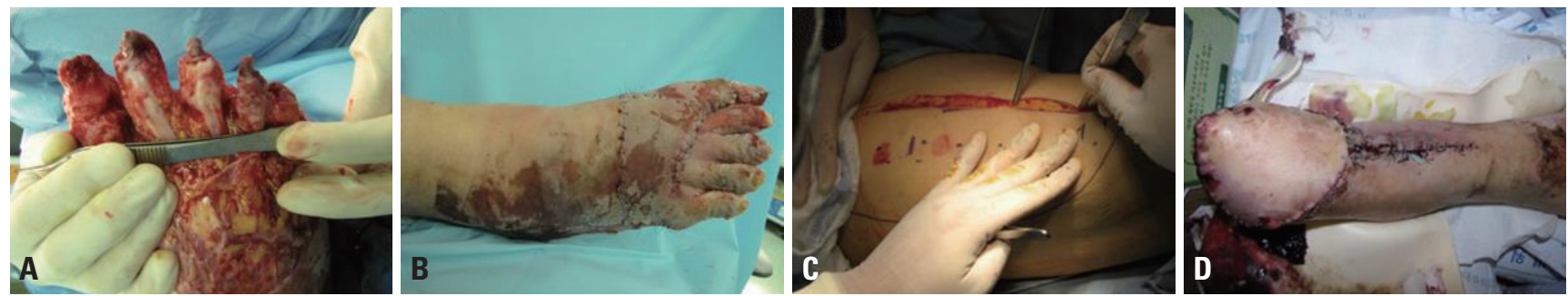

Fig. 1. (A) Degloving injury of the forefoot. (B) Immediate application of biologic dressing using stripped skin tissue. (C) Marking of the lateral circumflex femoral artery perforator branches with a handheld Doppler ultrasound device and elevation of the anterolateral thigh flap. (D) Postoperative focal congestion at the flap margin during the process of healing by secondary intention. 
between the descending branch of the lateral femoral circumflex artery and the perforation branch, dissection was performed in the proximal direction and the flap with a $10-\mathrm{cm}$-long pedicle was elevated. The lateral femoral cutaneous nerve that passes over the sartorius muscle and above the femoral fascia distally was included to make a

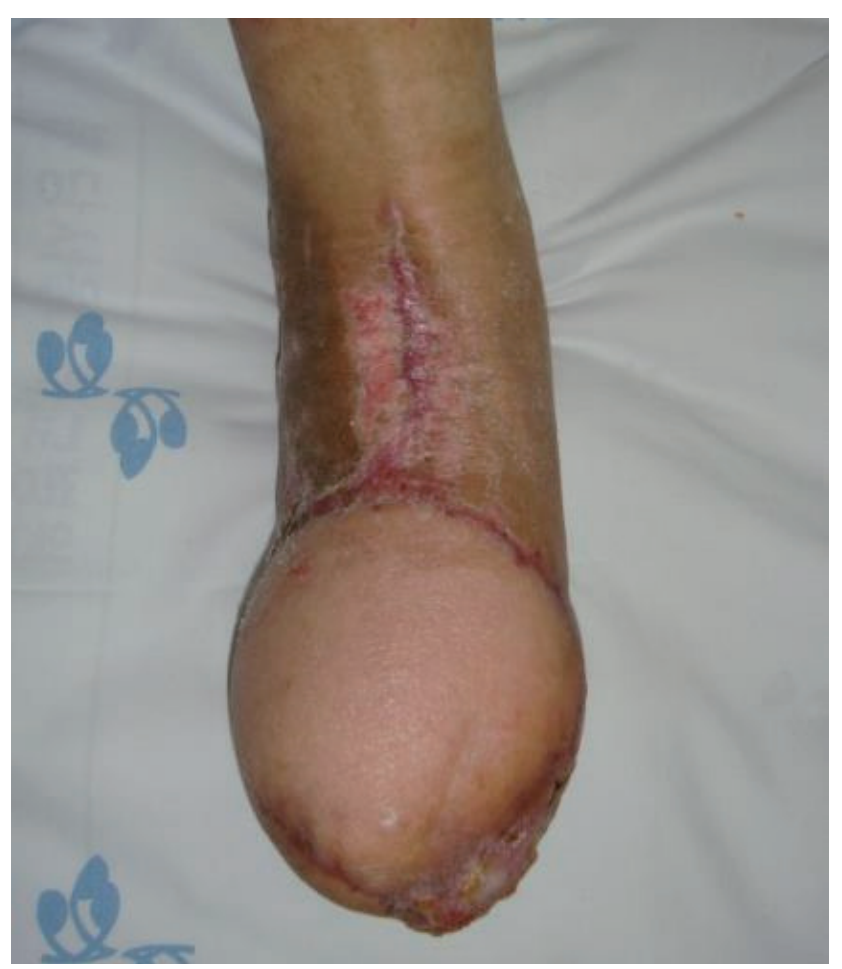

Fig. 2. At postoperative 10 months, the flap thickness was approximately 4-5 $\mathrm{mm}$ at the dorsal side and about 6-8 $\mathrm{mm}$ at the plantar side. sensate flap. After confirming the condition of the dorsalis pedis artery with the vena comitans in the pedicle at the recipient site of the forefoot and that at the donor site, the flap was positioned at the forefoot with a key suture. The forefoot defect was covered from the lateral side and positioned along the border, finally resulting in a complete syndactyly form of the foot. Under microscopy, end-toend anastomosis of the donor and recipient pedicles was performed, and the lateral femoral cutaneous nerve was coapted with the deep peroneal nerve. After confirming adequate circulation at the pedicle, closure between the flap margin and the defect was completed. At the postoperative follow-up, complications such as wound infection, hematoma, or necrosis of the flap were absent. Focal congestion was observed at the flap margin, but healing by secondary intention was achieved without additional surgical procedures (Fig. 1D). The suture was removed on postoperative day 14 , and the patient was discharged on postoperative day 25. The flap thickness measured at the immediate postoperative period was approximately $10 \mathrm{~mm}$, whereas it was about $4-5 \mathrm{~mm}$ at the dorsal area and $6-8 \mathrm{~mm}$ at the plantar area of the foot 10 months later (Fig. 2). In the static two-point discrimination test at 14 months postoperatively, the discrimination function was $11 \mathrm{~mm}$ at the dorsal area and 13-14 $\mathrm{mm}$ at the plantar area. In the moving two-point discrimination test, the discrimination function was $10 \mathrm{~mm}$ at the dorsal side and $11 \mathrm{~mm}$ at the plantar side. A debulking procedure was performed to achieve a shape and functionality that al-
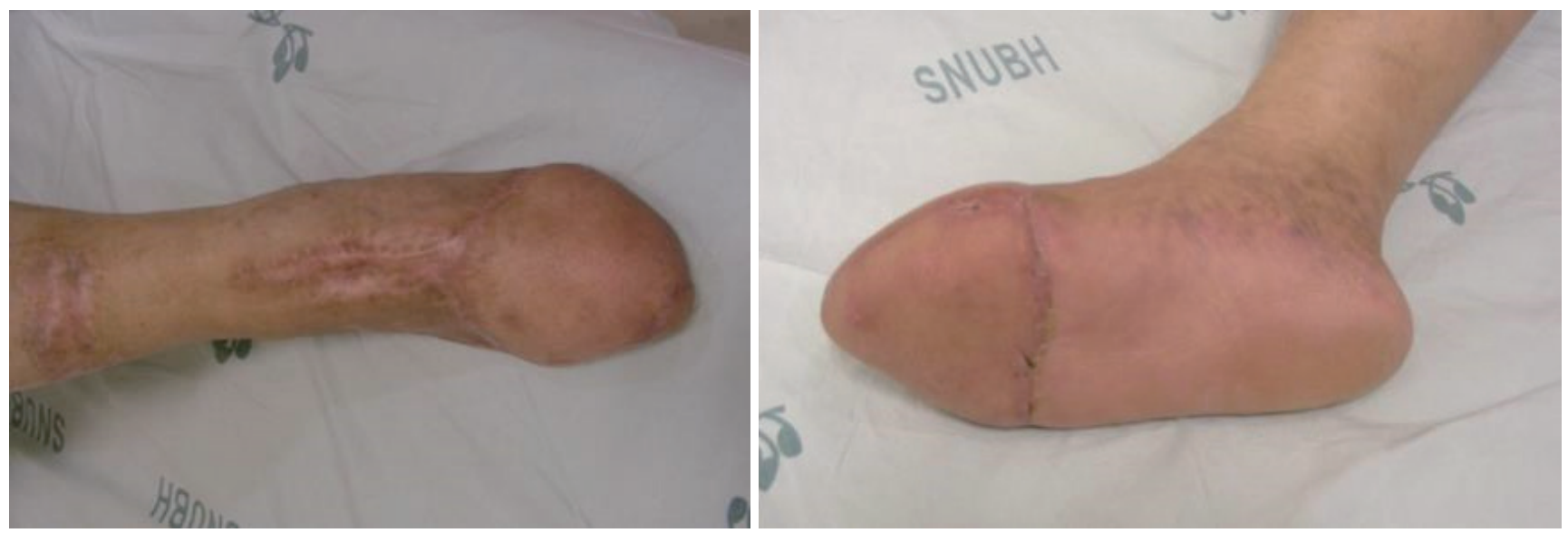

Fig. 3. Photograph of the foot at postoperative 6 months after the debulking procedure. 
lows wearing of proper shoe size and to improve comfort during walking. During the 14 months follow-up period, complications such as flap infection, ulceration, and flap loss were not observed. The patient was very satisfied with the shape and function of the reconstructed foot (Fig. 3).

\section{Case 2}

The patient was a 41-year-old man referred to department of plastic and reconstructive surgery for the treatment of open fracture of the distal phalanx of the big toe, with necrosis of the skin and soft tissue caused by a crushing injury of the foot (from a heavy dropped log) that occurred 2 weeks previously. On radiographs, multiple fractures of the distal phalanx of the big toe were noted. Primary marginal resection and $\mathrm{K}$-wire fixation were performed by the orthopedic surgeon; however, the fixation of the 1st distal phalanx was unstable, and progression of necrosis of the skin and soft tissue up to the proximal part of the hallux was observed (Fig. 4). The patient was relatively healthy without underlying diseases such as diabetes, hypertension, and vascular disease. At 2 weeks after the injury, reconstruction of the big toe with a free anterolateral thigh flap and iliac bone graft was planned. Under general anes- thesia, the necrotic skin and soft tissues were removed to expose the bone. Debridement was performed in the area up to the level of healthy marginal subcutaneous tissue. Multiple bony fragments of the fractured distal phalanx were removed, and an iliac bone portion of size $2 \times 1 \times 0.5 \mathrm{~cm}$ was harvested based on the size of the recipient bone. The shape of the bone was trimmed with burring and fixed firmly to the proximal portion by using a K-wire. The left anterolateral thigh flap of size $15 \times 6 \mathrm{~cm}$, which was slightly larger than the normal right big toe, was elevated using the conventional method as in the previous case, and primary closure of the donor site was completed. Under microscopy, end-to-end anastomosis of the vessel pedicle and both the dorsalis pedis artery and vena comitans was performed. The lateral femoral cutaneous nerve, which is the sensory nerve of the flap, was deeply coapted with the deep peroneal nerve. After the anastomosis, the flap was first positioned at the proximal portion of the big toe; the margins were partially trimmed, sutured along the border, and closure of the distal portion was finally completed. At the postoperative follow-up, complications such as wound infection, hematoma, and dehiscence of the donor site were absent, and focal congestion at the
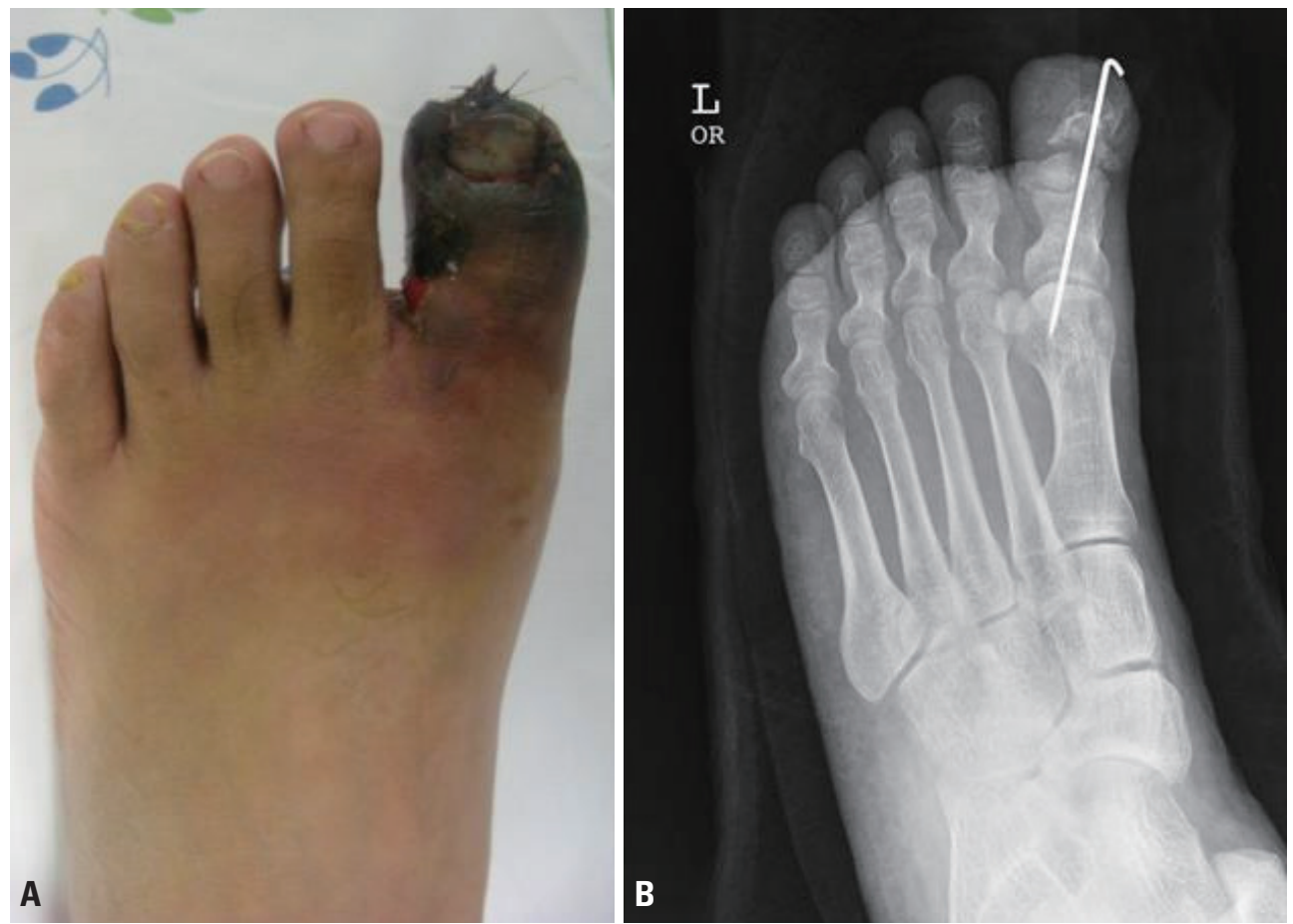

Fig. 4. (A) Necrosis of the skin and soft tissue in the left big toe. (B) Unstable fixation of the 1st distal phalanx. 
distal part of the flap and local partial necrosis were observed. The suture was removed on postoperative day 12 , and the patient was discharged on postoperative day 17 . The K-wire was removed 3 weeks postoperatively (Fig. 5A). Five months later, based on the shape and size of the normal right big toe, secondary flap reduction was performed (Fig. 5B, C). During the 24-month follow-up, the patient had normal walking ability with no specific discomfort. In addition, the patient was satisfied with the shape and size of the reconstructed big toe, which was similar to those of the contralateral big toe when wearing socks. In the twopoint discrimination test, a discrimination function of $12-15 \mathrm{~mm}$ was obtained, and some recovery of warm and cool sensation was achieved.

\section{DISCUSSION}

Several reports have described reconstruction methods using various tissues of the body in patients with degloving injury of the hand due to accidents $[1,2]$. However, degloving injury of the forefoot is relatively rare compared with that of the hand. The forefoot has a special anatomical structure, which poses difficulty for recon- structive surgeons in that many factors need to be considered, including weight-bearing function, stability, sensory function, and external shape of the forefoot. The forefoot has an important role in weight bearing and walking. If the bone or tendon is not exposed at the defect site, a skin graft may be primarily considered; however, forefoot defects are usually contraindicated for skin grafting and the simple skin graft cannot withstand weight bearing $[5,6]$. Previous reports have described the application of autologous dermal matrix and split thickness skin graft using degloved tissue in patients with bone exposure [5,7]. However, treatment for the dorsal surface is limited as compared with that for the forefoot or plantar surface, which is responsible for weight bearing. The free flap has the disadvantages of a long operation time, long-term hospitalization, and the need for a specialized technique for microvascular anastomosis. Nevertheless, it has strong advantages of good blood supply and sufficient tissue-volume replacement with autologous tissue. Moreover, reconstruction of the free flap provides durable tissue that can withstand weight loading, a rigid attachment that can overcome shearing force, and adequate protective sensation.

There are many free perforator flaps that can be used
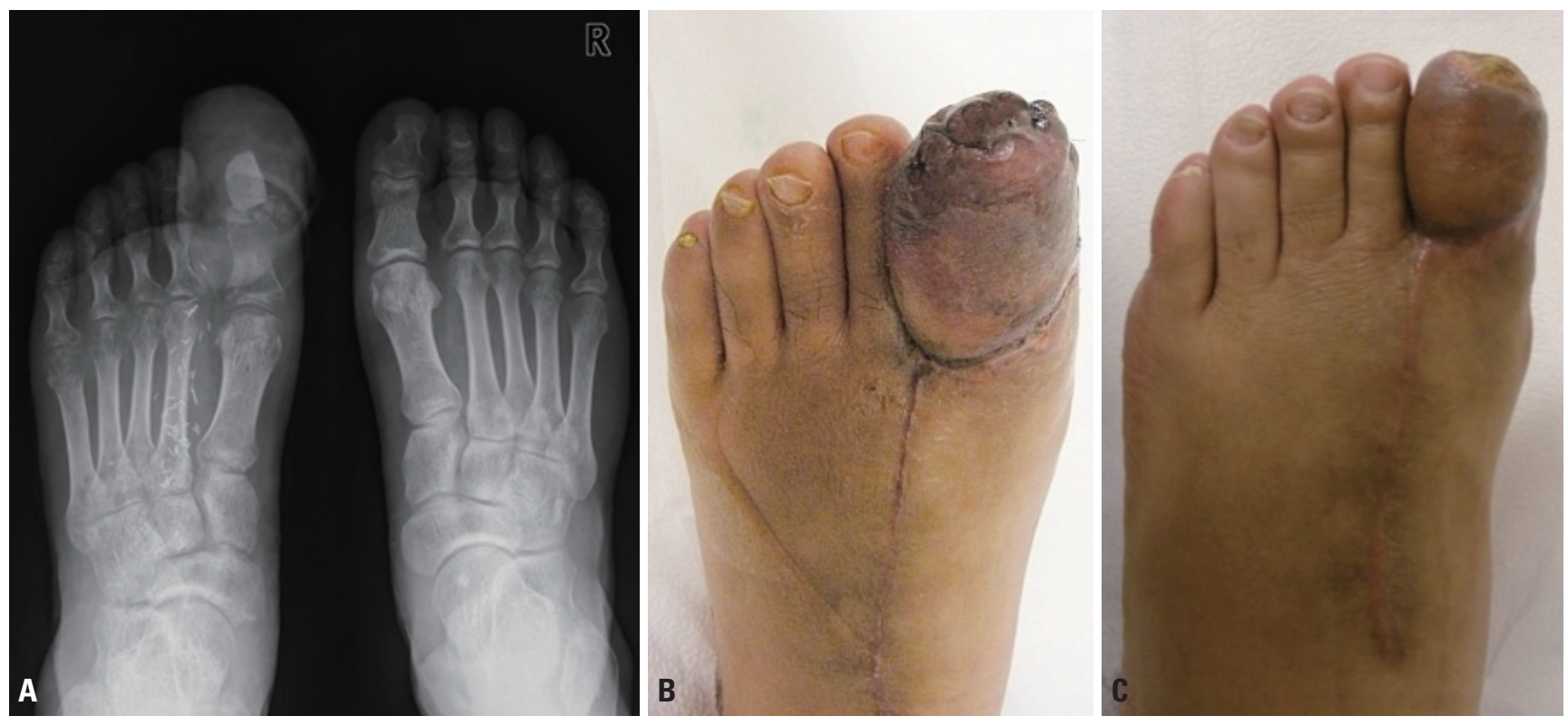

Fig. 5. (A) The K-wire was removed at 3 weeks postoperatively. (B) Preoperative photograph before the secondary flap debulking procedure. (C) Postoperative 24 months photograph of the debulked flap. 
as thin flaps, such as the superficial circumflex iliac artery perforator (SCIP) flap, medial sural artery perforator (MSAP) flap, and thoracodorsal artery perforator (TDAP) flap. The SCIP flap can be harvested as a thin flap with little or no muscle dissection, and the scars can be easily covered with a primary closure, resulting in low donor-site morbidity. However, the variation of the anatomical location of the superficial circumflex iliac artery is relatively large and the diameter of the pedicle is also variable and small. In addition, the short pedicle length, recipient-to-donor pedicle discrepancy, and donor-site complications such as lymphorrhea remain as problems [8]. The MSAP flap was first introduced in 2001 by Cavadas et al. [9]. Since then, this flap has evolved with various clinical applications. This flap is capable of muscle sparing, thus reducing donor-site morbidity, and can be elevated with various designs. A relatively long and large diameter perforator pedicle can be used. In addition, it is possible to reduce the risk of trimming in obese patients. However, because intramuscular dissection is necessary, the procedure becomes tedious and care must be taken to preserve a minute perforator that can be easily injured. The variation of the pedicle and skin graft for the donor site are considered to be the main disadvantages. Furthermore, it seems to have a higher risk of venous thrombosis than other established flaps [10]. The TDAP flap has a relatively long pedicle and a wide skin territory, making it a versatile flap. It can be used as a perforator flap and a muscle flap, as well as a chimeric flap for reconstructing a wide range of defects. However, its main disadvantage is that a septocutaneous perforator of adequate size is not always present. In these cases, it is inevitable to switch to a musculocutaneous flap [11]. In addition, the reliable size is still not established and the flexibility of the flap is insufficient owing to the thick skin; thus, it is not appropriate for contouring to a recipient site where circumferential reconstruction is required, as in our case. An ideal flap should have maximum possible thinness (as in the fasciocutaneous flap), low donor-site morbidity, and a long pedicle. Moreover, it should allow maintaining the same patient position intraoperatively. The radial forearm free flap is not suitable for reconstruction of the big toe because of donor-site morbidity and relatively more severe scar formation. The scapular or parascapular free flap requires changing the patient's posture intraoperatively, as well as additional dissection at the dorsal side. The fibular free flap and lateral arm free flap have the disadvantages of difficult closure of the primary donor site when a large flap size is required and obvious visibility of the donor site.

The wide dimension and relative pliability of the anterolateral thigh flap enables sufficient and adequate coverage of the injured and exposed structures of the foot. In addition, it is possible to achieve a flap skin thickness of $3-4 \mathrm{~mm}$ by properly removing the fat layer, and the large dead space can be covered by sufficient volume of the surrounding muscle if necessary. The sensory flap obtained by including a branch of the lateral femoral cutaneous nerve can achieve a faster recovery rate with protective sensation, lower risk of flap loss, and rapid rehabilitation and functional recovery compared with a non-sensory flap. In the first case, the patient could walk with a cane immediately after surgery and showed gradual sensory recovery. In addition, the plantar surface of the flap was stabilized. Finally, the patient achieved close to normal walking ability, and the overall size and shape of the foot were similar to those of the contralateral foot. The patient expressed satisfaction with the result. In the second case, the reconstruction of the big toe, which is considered the most important part of the forefoot, was successful. The patient was relatively young and strongly desired reconstruction of his big toe.

Surgeons should perform reconstruction of the big toe while considering functional aspects such as normal walking and wearing of proper shoe size, as well as the aesthetic outcome. An optimum reconstruction method considers the cause and size of the defect, defect site and condition of the surrounding tissue, availability of the donor site, and postoperative normal walking ability. Currently, many methods for reconstruction of the big toe are available, including pedicle flaps such as the reversed dorsalis pedis flap, reversed tibialis anterior flap, reversed medial plantar flap, medial plantar flap, and reversed dorsal metatarsal flap [12-14]. However, extensive necrosis of the skin and subcutaneous tissues may occur, and surgeons should completely cover the grafted iliac bone, which requires a large amount of soft tissue. In such cases, reconstruction of the big toe with the pedicle flap is limit- 
ed, and operators should consider reconstruction with the free flap.

The anterolateral free flap has low donor-site morbidity, allows primary closure even in cases that require a large flap, and eliminates the need for changing the patient's position. The big toe requires a large amount of tissue. In our case, a $360^{\circ}$ coverage of the grafted iliac bone required a sufficiently large flap. Considering the anastomosis of the vessel and the stability and shape, the flap should preferably cover the bone transversely, with the suture line located on the side. In the secondary flap reduction procedure, we planned to refine the shape of the dorsal aspect of the forefoot. The overall size and shape after the postoperative secondary procedure were remarkably similar to those of the contralateral big toe, and the patient was satisfied with the results.

In conclusion, the sensate anterolateral thigh flap is a useful and reliable reconstruction option for big toe and forefoot defects. Reconstruction of the forefoot and big toe should be performed with consideration of the important roles of these body parts in walking, standing, maintaining balance, and supporting the body when changing directions. Surgeons should attempt active reconstruction in appropriately indicated cases while considering the cultural specificities of daily living activities without wearing shoes.

\section{REFERENCES}

1. Krishnamoorthy R, Karthikeyan G. Degloving injuries of the hand. Indian J Plast Surg 2011;44:227-36.

2. Zhang G, Ju J, Jin G, Tang L, Fu Y, Hou R. Replantation or revascularization for the treatment of hand degloving injuries. J Plast Reconstr Aesthet Surg 2016;69:1669-75.

3. Smith BR, Begeman PC, Leland R, Meehan R, Levine RS, Yang
$\mathrm{KH}$, et al. A mechanism of injury to the forefoot in car crashes. Traffic Inj Prev 2005;6:156-69.

4. Zgonis T, Cromack DT, Stapleton JJ. Utilizing a crossover reverse sural artery flap for soft tissue reconstruction of the plantar forefoot after a severe degloving injury. Int J Low Extrem Wounds 2007;6:114-9.

5. Huemer GM, Schoeller T, Dunst KM, Rainer C. Management of a traumatically avulsed skin-flap on the dorsum of the foot. Arch Orthop Trauma Surg 2004;124:559-62.

6. Yeh JT, Lin CH, Lin YT. Skin grafting as a salvage procedure in diabetic foot reconstruction to avoid major limb amputation. Chang Gung Med J 2010;33:389-96.

7. Hong JP, Kim EK. Sole reconstruction using anterolateral thigh perforator free flaps. Plast Reconstr Surg 2007;119:186-93.

8. Kim JH, Kim KN, Yoon CS. Reconstruction of moderate-sized distal limb defects using a superthin superficial circumflex iliac artery perforator flap. J Reconstr Microsurg 2015;31:631-5.

9. Cavadas PC, Sanz-Giménez-Rico JR, Gutierrez-de la Cámara A, Navarro-Monzonís A, Soler-Nomdedeu S, Martínez-Soriano F. The medial sural artery perforator free flap. Plast Reconstr Surg 2001;108:1609-15; discussion 1616-7.

10. Toyserkani NM, Sørensen JA. Medial sural artery perforator flap: a challenging free flap. Eur J Plast Surg 2015;38:391-6.

11. Miyamoto S, Arikawa M, Kagaya Y, Fukunaga Y. Septocutaneous thoracodorsal artery perforator flaps: a retrospective cohort study. J Plast Reconstr Aesthet Surg 2019;72:78-84.

12. Cheng MH, Ulusal BG, Wei FC. Reverse first dorsal metatarsal artery flap for reconstruction of traumatic defects of dorsal great toe. J Trauma 2006;60:1138-41.

13. Niranjan NS, Vanstralen P. Homodigital reverse pedicle island flap for reconstruction of the great toe. Br J Plast Surg 2000;53:499-502.

14. Balakrishnan C, Chang YJ, Balakrishnan A, Careaga D. Reversed dorsal metatarsal artery flap for reconstruction of a soft tissue defect of the big toe. Can J Plast Surg 2009;17:e11-2. 